\title{
HPV vaccination: what should we know as otorhinolaryngologists?
}

Keywords: HPV vaccination, oropharynx, oropharyngeal cancer

\section{Introduction}

Human papillomaviruses (HPV) infection is the most frequent sexually transmitted infection worldwide. HPV are ubiquitous viruses that infect epithelial tissue of males and females. ${ }^{1,2}$

More than 150 HPV genotypes have been identified and approximately 40 of them can infect mucosal genital, oral and oropharyngeal epithelial cells. According to their oncogenic potential (established to uterine cervix cancer) they are divided in high risk (HR) and low risk (LR) genotypes. ${ }^{1-3}$

Most people will be infected with HPV at least one time in their lifetime. The infection is usually asymptomatic and cleared by the immune system. Sometimes, the infection becomes persistent, leading to benign, premalignant ou malignant lesions, depending on HPV genotype oncogenic potential (high risk vs low risk), and host immune status. ${ }^{1-3}$

Although it's causative role in cervix cancer is well known, HPV has been implicated in several other human cancers such as in head and neck region, specially the oropharynx, where mostly $50 \%$ of cases are associated to HPV (subgroup of HPV positive oropharyngeal cancer). ${ }^{4}$

There's lack of epidemiological data on oropharyngeal HPV infection, although it's incidence is assumed to be rising..$^{5-8}$ In a US populational-based study, oral HPV infection prevalence was found to be $6,8 \%$ in both genders, between 14 and 69 years old, with HPV 16 responsible for $1 \%$ of the cases. ${ }^{5}$

HPV infection is transmitted sexually by close contact, even in the absence of penetration, by vaginal, anal and oral intercourse. In the oropharyngeal HPV infection, HPV is also transmitted sexually trough oral sex; rarely, it can also be acquired from vertical transmission or auto-inoculation. ${ }^{1-3}$

Rising numbers of HPV positive oropharyngeal cancer makes relevant the discussion about HPV carcinogenesis in the oropharynx. ${ }^{4}$ There is a subgroup of patients with oropharyngeal cancer, HPV positive and located preferentially in palatine or lingual tonsils. When compared to the HPV negative patients, they are younger, not exposed to alchool or tobacco, have lower $\mathrm{T}$ and higher $\mathrm{N}$ at initial TNM staging, are more radiosensitive and have better vital prognosis. ${ }^{6}$

\section{Could we prevent HPV related oropharyngeal cancer? \\ Prevention}

HPV infection is preventable. ${ }^{1-3}$ As the infection manifests itself in the ENT territory, all otorhinolaryngologists should be aware of its prevention measures for proper patient advise.

\author{
Volume II Issue I - 2019
}

\author{
Jardim A,' Fraga T, ${ }^{2}$ Montalvao P,, 3 Marinho \\ M,' Saraiva J' \\ 'Otorhinolaryngology Department, CUF Descobertas Hospital, \\ Portugal \\ ${ }^{2}$ Ginaecology and Obstetrics Department, CUF Descobertas \\ Hospital, Portugal \\ ${ }^{3}$ Otorhinolaryngology Department, Portuguese Oncological \\ Center, Instituto Portugues de Oncologia de Lisboa, Francisco \\ Gentil, Portugal
}

\section{Correspondence: Ana Jardim, ENT Resident at CUF} Descobertas Hospital, Otorhinolaryngology Department, Rua Mario Botas, Parque das Nacoes, 1998-018 Lisboa, Portugal, Tel +351 210025 200, Email anacristinajardim@gmail.com

Received: August 12, 2018| Published: January 07, 2019

\section{Primary prevention}

\section{Avoid risk factors}

a. Identify and modify behavior risk factors: reduce sexual partners number (since prevalence of infections rises with the numbers of sexual partners)

b. Use condom at sexual intercourse (although this measure is not completely effective, since it doesn't cover all the skin at genital region, it reduces chances of being infected). ${ }^{2,3}$

\section{Vaccination}

Acquisition of HPV infection can be avoid through prophylactic vaccination. There are actually 3 anti-HPV prophylactic vaccines: they are non-infectious recombinant vaccines, prepared from the purified VLP (vírus-like particules) of the major capsid (L1) protein of HPV; each vaccine covers specific HPV genotypes as follows:

1. Bivalent HPV vaccine (2vHPV), (Cervarix ${ }^{\circledR}$, GlaxoSmithKline) includes $2 \mathrm{HR}-\mathrm{HPV}$ types, 16 and 18.

2. Quadrivalent HPV vaccine, $4 \mathrm{vHPV}$ (Gardasil $\AA$, Merck Sharp \& Dohme Corp.): includes 4 HPV types, 16 and 18 (HR), 6 and 11 (LR).

3. Nonavalent HPV vaccine, 9vHPV (Gardasi19 $®$, Merck Sharp \& Dohme Corp.): includes 4 types from the quadrivalent vaccine (16, $18,6,11$ ), plus 5 HR types, $31,33,45,52$, e 58 .

4. According to CDC and Advisory Committee on Immunization Practices, HPV vaccination is recommended in the following cases:

A. Routine HPV Vaccinations for adolescents (boys and girls) at age 11 or 12 years. 
B. For people who did not start or complete vaccination when they were younger, vaccination is also recommended for:

a. Males ages 13 through 21 years

b. Females ages 13 through 26 years

c. Gay, bisexual, and other men who have sex with men, transgender people

d. And persons with certain immunocompromising conditions ages 22 through 26

The ideal timing for vaccination is in adolescence, prior to sexual activity and exposure to HPV (HPV-naive status). However, people who already initiated sexual activity, or have already been infected with one or more HPV types, can still benefit from vaccine protection against the other types included in the vaccine.

Before 15 years old (or HPV naive status), a 2-dose vaccine schedule is recommended, with the second dose $6-12$ months after the first one ( $0,6-12$ month schedule). For all the other a 3-dose schedule is recommended $\left(0,1-2,6\right.$ month schedule)..$^{9,10}$

In Portugal, HPV vaccination is present in National Health Immunization Program (Plano Nacional de Vacinação - PNV) since 2008, but it was only after 2014 that HPV vaccination was recommended to all girls at age 10 years old, in a 2-dose schedule. ${ }^{11}$ Universal immunization, meaning, including boys in the program, is actually being publically discussed, although it's already recommended for the Portuguese Pediatric Society. ${ }^{12}$

Persistent high-risk HPV infection can cause cervical, vaginal and vulvar cancer in women; penile cancer in men, and anal and oropharyngeal cancer in both men and women. $80 \%$ of HPV related cancer is caused by HR genotypes 16 and 18, included in $2 \mathrm{vHPV}$, $4 \mathrm{vHPV}$ e 9vHPV vaccines.

Low-risk HPV infection can cause benign lesions, such as genital warts or rarely, laryngeal papillomas; they can also cause benign or low-grade cervical cell abnormalities. Almost all genital warts and papillomas are caused by genotypes 6 and 11, included in 4vHPV e 9vHPV vaccines.

HPV vaccines are safe. They went through years of safety testing before were licensed by the U.S. Food and Drug Administration (FDA). Several thousands of people were studied in clinical trials before vaccines were licensed: 15000 males and females for 9vHPV vaccine, 29000 males and females for 4vHPV vaccine and 30000 females for $2 \mathrm{vHPV}$ vaccine. Their safety is still monitored with more than 100 million doses distributed in the United States with reassuring safety records., ${ }^{9,10}$

Regarding HPV vaccines efficacy in cancer prevention, talking specifically of $9 \mathrm{vHPV}$ vaccine, its efficacy is demonstrated for preventing cervical, vaginal, vulvar, anal and penile cancer. For oropharyngeal cancer, there's still lacking studies demonstrating it's efficacy. Nonetheless, there are more and more studies that proved HPV vaccine efficacy in:

i. Reduction oral HPV infection ${ }^{5,13}$

ii. Immunological response with specific antibodies production (in relation to the types included in the vaccine) in serum and saliva $^{14-16}$
If we take into account that HPV positive oropharyngeal cancer is mostly related to genotypes 16 and 18 , which are included in all HPV vaccines, and that vaccination is effective in preventing oral/ oropharyngeal infection, would be reasonable to think that vaccination would reduce incidence of oropharyngeal cancer related to HPV infection.

HPV vaccines are also effective in prevention of genital warts, as long as they include genotypes 6 and 11 (4vHPV and 9vHPV). Regarding laryngeal papillomatosis, studies show that it can benefit from prophylactic vaccines $4 \mathrm{vHPV}$ e $9 \mathrm{vHPV}$, but only in inhibiting lesions recurrence. ${ }^{15}$

One thing to consider in all HPV lesions is that HPV prophylactic vaccines do not treat any existing lesion; they are meant to prevent acquiring future HPV infection.

Therapeutical vaccines, in the other hand, are meant to treat existing lesions, but for now, are used only in investigation settings. One example is an animal studie using a therapeutical vaccine directed to E6E7 proteins from an HPV11 tumor; it showed T-CD8+ cellular response capable of reducing HPV11 tumor growth. ${ }^{17}$ This results are promising and should be exciting for ENT doctors, since it shows reduced tumor growth on HPV 11 lesions, the genotype related to worse laryngeal papillomatosis severity and prognosis. This vaccine could become a therapeutical weapon for these difficult cases in a near future.

In ENT context, there is no consensus recommending prophylatic HPV vaccination. Nevertheless, regarding the favorable results of $\mathrm{HPV}$ vaccination on reducing oral HPV infection, and, on inhibiting laryngeal papillomatosis recurrence, HPV vaccines could be recommended individually, accordingly to:
a. Patients risk behavior
b. Patients immune status
c. ENT expert opinion
d. Patients choice

HPV infection does not cause, in ENT regions, an HPV lesion identifiable as precursor of HPV positive oropharyngeal cancer (contrary to what happens in uterine cervix), so there is no role for screening.

Regarding contacts, there is also no recommendation to screening sexual contacts for HPV, although is common sense that sexual partners with active HPV lesions, either oral or genital, should be seen by a doctor and offered treatment.

\section{Secondary prevention: early diagnosis}

It was not identified yet an HPV lesion considerd precursor os oropharyngeal cancer, so population screening programs designed to identify HPV premalignat lesions is neither usefull or cost-effective for HPV positive oropharyngeal cancer.

It means that early diagnosis is still the key measure when talking about secondary prevention. All oropharyngeal lesions that do not subside within 2-3 weeks besides proper treatment should be investigated, and cancer should be ruled out. According to NCCN2017 recommendations, all oropharyngeal tumors should be HPV tested. $^{18}$ 


\section{Author's note}

There is still a lot to learn about HPV infection and natural history in ENT territory. Nonetheless, a rising number of oropharyngeal cancer cases are linked to HPV. Since HPV infection is preventable trough vaccination, and since HPV vaccines are available, effective and safe, we should rethink the benefits of recommending HPV prophylatic vaccines to ENT patients.

\section{Acknowledgements}

None.

\section{Conflict of interest}

Authors declare that there is no conflict of interest.

\section{Funding details}

None.

\section{References}

1. IARC Monographs on the Evaluation of Carcinogenic Risks to Humans. Human Papillomaviruses. 2012;100B.

2. Human Papillomavirus (HPV) For Clinicians. 2018.

3. HPV. Liga Portuguesa Contra o Cancro. 2018.

4. Betiol J, Villa LL, Sichero L. Impact of HPV infection on the development of head and neck cancer. Braz J Med Biol Res. 2013;46(3):217-226.

5. Gillison ML, Broutian T, Pickard RK, et al. Prevalence of oral HPV infection in the United States, 2009-2010. JAMA. 2012;307(7):693703.

6. Psyrri A, Cohen E. Oropharyngeal cancer: clinical implications of the HPV connection. Annals of Oncology. 2011;22(5):997-999.

7. Mravak-Stipetic M, Sabol I, Kranjcic J, et al. Human Papillomavirus in the Lesions of the Oral Mucosa According to Topography. PLoS ONE. 2013;8(7):e69736
8. Castro TPPG, Filho IB. Prevalence of human papillomavirus (HPV) in oral cavity and oropharynx. Braz J Otorhinolaryngol. 2006;72(2):272282.

9. HPV Vaccine Information for Clinicians. 2018.

10. Vaccination Schedules \& Recommendations. 2018.

11. Programa Nacional de vacinação - 2017. Direcção Geral de Saúde. Junho, 2017.

12. Recomendações sobre vacinas extra programa nacional de vacinação actualização 2017 - Comissão de Vacinas da Sociedade de Infeciologia Pediátrica e da Sociedade Portuguesa de Pediatria - Julho, 2017.

13. Herrero R, Quint W, Hildesheim A, et al. Reduced Prevalence of Oral Human Papillomavirus (HPV) 4 Years after Bivalent HPV Vaccination in a Randomized Clinical Trial in Costa Rica. PLoS ONE. 2013;8(7):e68329.

14. Chaturvedi AK, Graubard BI, Broutian T, et al. Effect of Prophylactic Human Papillomavirus (HPV) Vaccination on Oral HPV Infections among Young Adults in the United States. J Clin Oncol. 2018;36(3):262267.

15. Makiyama K, Hirai R, Matsuzaki H, et al. Gardasil vaccination for recurrent laryngeal papillomatosis in adult men: first report: changes in HPV antibody titer. J Voice. 2017;31(1):104-106.

16. Pinto LA, Kemp TJ, Torres BN, et al. Quadrivalent Human Papillomavirus (HPV) Vaccine induces HPV-specific antibodies in the oral cavity: results from the Mid-Adult Male Vaccine Trial. J Infect Dis. 2016;214(8):1276-1283.

17. Ahn J, Peng S, Hung C, et al. Immunologic responses to a novel DNA vaccine targeting human papillomavirus 11 E6E7. The Laryngoscope. 2017;127(12):2713-2720.

18. NCCN Clinical PracticeGuidelines in Oncology (NCCN Guidelines $\left.{ }^{\circledR}\right)$. Head and Neck Cancers. 2017. 